\title{
Effectiveness of Physical and Psychological Treatment for Cancer-Related Fatigue: Systematic Review
}

\author{
Dian Hudiyawati ${ }^{1 *}$, Wulan Syafitry ${ }^{2}$ \\ ${ }^{1}$ School of Nursing, Faculty of Health Science, Universitas Muhammadiyah Surakarta, \\ Surakarta, Central Java, Indonesia \\ "Corresponding author \\ ${ }^{2}$ Student School of Nursing, Faculty of Health Science, Universitas Muhammadiyah Surakarta \\ Email: ${ }^{1}$ dian.hudiyawati@ums.ac.id; ${ }^{2}$ 210174134@ student.ums.ac.id
}

Submission Date: August 27, 2021 ; Acceptance Date: December 01, 2021

\begin{abstract}
Cancer is the second leading cause of death worldwide. Fatigue is one of the most common side effects of people with cancer. The range of people with cancer who experience fatigue is $70-80 \%$, when undergoing cancer treatment and during the phase before and after treatment. This systematic review aims to identify fatigue management with non-pharmacological intervention. The journals selected from 2015 to 2020 in an international database: Pubmed, SAGE journals, Microsoft Academic, and Science Direct. The database searched using the keywords "exercise treatment" OR "psychological treatment," AND "fatigue" OR "cancer-related fatigue," AND "during cancer treatment" OR "after cancer treatment." Assessment of Critical quality appraisal uses tools from the JBI (Joanna Briggs Institute) critical assessment checklist. The method of analysis used the descriptive method. The results of the Systematic review found 13 journals that met the inclusion criteria, the total sample was 1365 respondents with an average age of 56.85, and the consequences of female respondents were 857 respondents, and male respondents were 464 respondents. Physical treatment and psychological treatment can reduce cancer fatigue before and after treatment. The interventions given both physical treatment and psychological treatment have their benefits and vary in their effectiveness.
\end{abstract}

Keywords: cancer-related fatigue, fatigue, physical treatment, psychological treatment

ISSN 1979-7621 (Print). ISSN 2620-7761 (Online).

DOI 10.23917/jk.v14i2.15596

\section{INTRODUCTION}

Cancer is the second leading cause of death worldwide, based on data from the Global Cancer Observatory (Globocan) in 2018, the number of new cancer cases was more than 18 million cases, and the number of deaths was more than 9 million deaths (Akaza, 2019). Based on data from Basic Health Research (Riskesdas) in 2018, there was an increase in the prevalence of Indonesian cancer based on 2013 , is $1.4 \%$ to $1.8 \%$ in 2018 , Central Java is in the tenth position with a 
prevalence of $2.1 \%$ and there is no significant increase or decrease between 2013 and 2018 (Riskesdas, 2018). Fatigue is one of the most common side effects of people with cancer. The range of cancer patients who experience fatigue is $70-80 \%$. Among other things, when undergoing radio treatment, chemo treatment and cancer treatment, and during the phase before and after treatment (Charalambous et al., 2019).

Research shows that cancer patients who experience fatigue can impact the activities of daily life and affect the quality of life of cancer patients. Besides, cancer patients who experience continuous fatigue have a higher risk of death than patients who have never experienced fatigue (the risk of death reaches 2.56 times). Fatigue also has a significant impact on patients' working lives, based on $75 \%$ of 177 patients reporting have changed their employment status due to fatigue (Aapro et al., 2017).

The results showed that physical exercise, psychological treatment, and physical exercise with psychological therapeutic interventions effectively reduced cancer-induced fatigue during and after primary care, while pharmacological interventions were ineffective in reducing cancer-induced fatigue. Physical exercise interventions and psychological treatment were significantly more effective at reducing fatigue levels than overall pharmacological interventions (Mustian et al., 2017). Although physical exercise and nonpharmacological treatment are effective in reducing cancer-induced fatigue (Hilfiker et al., 2018). Based on the description above, it is necessary to conduct a literature review that aims to identify fatigue management with nonpharmacological interventions such as physical exercise treatment and psychological treatment, which are most effective in managing fatigue due to cancer during cancer treatment and after cancer treatment.

\section{METHODS}

The writing design is a Systematic Review. The systematic review uses a protocol and evaluation, which is PRISMA, to determine the completion of studies found and adapted to the objectives of the systematic review.

\section{Data sources and screening process}

The data used in this research is secondary data obtained from the results of the study conducted by previous researchers, using four databases is: Pubmed, SAGE journals, Microsoft Academic, Science Direct. Searching for articles and journals in this review systematic review uses keywords and Boolean operators (AND, OR NOT or AND NOT) (Zohuri and Moghaddam 2017). Is by keywords "exercise treatment" OR "psychological treatment," AND "fatigue" OR "cancer-related fatigue," AND "during cancer treatment" OR "after cancer treatment." The strategy used to search for articles and journals is using the PICOS framework (Saaiq and Ashraf, 2017).

Table 1. Inclusion and exclusion criteria of the studies in the systematic review

\begin{tabular}{|c|c|c|}
\hline Criteria & Inclusion & Exclusion \\
\hline Patient Population & $\begin{array}{l}\text { Studies comprised affected } \\
\text { communities with Cancer }\end{array}$ & Communities not affected Cancer \\
\hline Intervention & Non-pharmaceutical & Pharmaceutical treatment \\
\hline
\end{tabular}


D Hudiyawati \& W Syafitry / Jurnal Kesehatan 14 (2) 2021, 195-211

\begin{tabular}{|c|c|c|}
\hline Criteria & Inclusion & Exclusion \\
\hline & $\begin{array}{l}\text { such as exercise treatment and } \\
\text { psychological treatment }\end{array}$ & \\
\hline Comparative controls & No comparative & \\
\hline Outcomes & $\begin{array}{l}\text { The effectiveness } \\
\text { nonpharmacological treatment for } \\
\text { cancer related fatigue }\end{array}$ & $\begin{array}{l}\text { Not described the effectiveness of } \\
\text { nonpharmacological treatment for } \\
\text { cancer related fatigue }\end{array}$ \\
\hline Statistical analysis & Randomized control and trial. & $\begin{array}{l}\text { Systematic review, meta-analyses, A } \\
\text { Quasi-experimental study, cross- } \\
\text { sectional studies. and qualitative } \\
\text { research }\end{array}$ \\
\hline Publication years & $2015-2020$ & Before-2015 \\
\hline Language & English & Language other than English \\
\hline
\end{tabular}

\section{Data extraction}

Based on the literature searches through publications in four databases and using keywords adjusted to the keywords and Boolean Operators, the researchers found 2664 articles that match these keywords. The search results obtained rechecked, the researcher then analyzes based on the title to get the abstract results reviewed with an assessment based on the eligibility of the inclusion and exclusion criteria, as many as 1711 articles not included in the outcomes criteria, 519 papers not included in the intervention criteria, 227 reports Not included in the standards for the patient population, 157 articles not included in the requirements for statistical analysis, 1 article found in Indonesian. Finally, there were 49 articles for abstract analysis.

Quality assessment
The analytical method used in this systematic review is a descriptive method based on a theme determined in the systematic review. The study uses descriptive analysis that describes and explains through tables and narratives about the research results described in the literature - data analysis methods using data extraction formats. Assessment of Critical quality appraisal uses tools from the JBI (Joanna Briggs Institute) critical assessment checklist. Assessment criteria score of "yes," "no," "unclear," and "not valid," each measure with the result of "yes" is given one point, and the score for the other criteria is zero, each score calculated and added up. If the results of the study at least $50 \%$ meet the Critical appraisal criteria with the cut-off point value agreed upon by the researcher, the study included in the inclusion criteria (Higgins et al., 2019). 


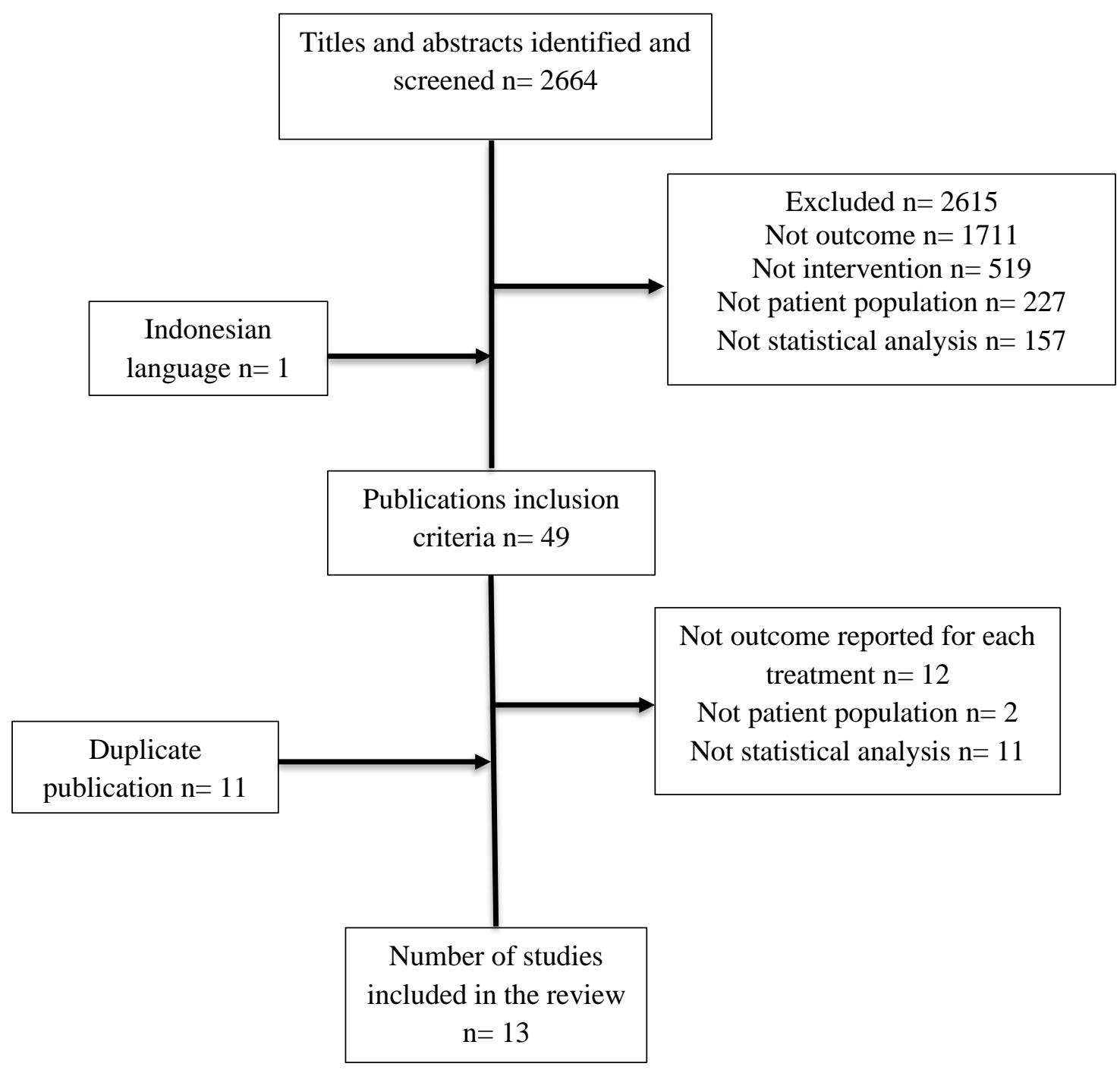

Figure 1. PRISMA Flow

The researcher then analyzed 49 articles based on abstract tracing and obtained 11 duplication articles, duplication analysis using Mendeley software with Check for Duplicates tools. The remaining 38 articles will be analyzed based on the abstract using a form consisting of problems, objectives, methods, and research results. The analysis results based on the abstract found 12 articles do not match the outcome criteria to be analyzed, two papers do not match the patient's requirements to explored, and 11 articles do not fit the criteria for statistical analysis. The results were 13 articles that continued into full text. 


\section{RESULTS AND DISCUSSION}

\section{Respondent characteristics}

Respondents in this study were all patients diagnosed with cancer in each country, with a total of 1564 respondents.
Respondents in this study had an average age of 40 to 67.9, with an average age of all respondents is 56.85. The gender characteristics of the respondents in this study were more female respondents than male respondents, with the results of female respondents totaling 857 respondents and male respondents totaling 464 respondents.

Table 2. Characteristic of the Study

\begin{tabular}{|c|c|c|c|c|c|c|c|c|c|c|c|c|c|c|c|}
\hline No & Component & $\begin{array}{l}\text { (Lin } \\
\text { et al. } \\
2019 \text { ) }\end{array}$ & $\begin{array}{l}\text { (Amy J. } \\
\text { Hoffman } \\
\text { et al. } \\
2017)\end{array}$ & $\begin{array}{l}\text { (Schuler } \\
\text { et al. } \\
2017)\end{array}$ & $\begin{array}{l}\text { (Lu } \\
\text { et al. } \\
2019)\end{array}$ & $\begin{array}{l}\text { (Bryant } \\
\text { et al. } \\
2018 \text { ) }\end{array}$ & $\begin{array}{l}\text { (Dhillon } \\
\text { et al. } \\
\text { 2017) }\end{array}$ & $\begin{array}{l}\text { (Lundt } \\
\text { and } \\
\text { Jentschke } \\
\text { 2019) }\end{array}$ & $\begin{array}{l}\text { (Zhang } \\
\text { et al. } \\
\text { 2016) }\end{array}$ & $\begin{array}{l}\text { (Hojan, } \\
\text { Kwiatkowska- } \\
\text { Borowczyk, et } \\
\text { al. 2016) }\end{array}$ & $\begin{array}{l}\text { (Poort } \\
\text { et al. } \\
2020)\end{array}$ & 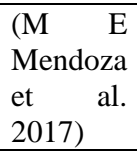 & $\begin{array}{l}\text { (Charalambous } \\
\text { et al. 2016) }\end{array}$ & $\begin{array}{l}\text { (Johns } \\
\text { et al. } \\
2015)\end{array}$ & Result/mean \\
\hline 1 & $\begin{array}{l}\text { Demographics } \\
\text { of data }\end{array}$ & 358 & 72 & 70 & 77 & 17 & 111 & 58 & 91 & 54 & 134 & 44 & 208 & 71 & 1365 \\
\hline 2 & Age (average) & 54,3 & 67 & 52,38 & 54,63 & 40,4 & 64 & 58,19 & 60 & 67,9 & 62,71 & 60,95 & 40 & 56,6 & 56,85 \\
\hline 3 & $\begin{array}{l}\text { Gender } \\
\text { Male }\end{array}$ & 14 & 32 & 41 & 56 & 12 & 61 & 6 & 68 & - & 57 & 5 & 104 & 8 & 464 \\
\hline & Female & 344 & 40 & 29 & 31 & 5 & 50 & 52 & 23 & - & 77 & 39 & 104 & 63 & 857 \\
\hline 4 & $\begin{array}{l}\text { Job Status } \\
\text { Work }\end{array}$ & 292 & 20 & - & - & - & - & - & - & - & 41 & 18 & - & 35 & 406 \\
\hline & Retired & - & 40 & - & - & - & - & - & - & - & 54 & 5 & - & - & 99 \\
\hline & Does not work & - & 12 & - & - & - & - & - & - & - & 31 & 21 & - & - & 64 \\
\hline & Other & - & - & - & - & - & - & - & - & - & 8 & - & - & 36 & 44 \\
\hline 5 & $\begin{array}{l}\text { Types of cancer } \\
\text { Ginekologi }\end{array}$ & 291 & - & 6 & - & - & - & 36 & - & - & 60 & 29 & 104 & 34 & 560 \\
\hline
\end{tabular}


D Hudiyawati \& W Syafitry / Jurnal Kesehatan 14 (2) 2021, 195-211

\begin{tabular}{|c|c|c|c|c|c|c|c|c|c|c|c|c|c|c|c|}
\hline No & Component & $\begin{array}{l}\text { (Lin } \\
\text { et al. } \\
2019 \text { ) }\end{array}$ & $\begin{array}{l}\text { (Amy J. } \\
\text { Hoffman } \\
\text { et al. } \\
2017)\end{array}$ & $\begin{array}{l}\text { (Schuler } \\
\text { et al. } \\
2017)\end{array}$ & $\begin{array}{l}\text { (Lu } \\
\text { et al. } \\
2019)\end{array}$ & $\begin{array}{l}\text { (Bryant } \\
\text { et al. } \\
2018 \text { ) }\end{array}$ & $\begin{array}{l}\text { (Dhillon } \\
\text { et al. } \\
2017 \text { ) }\end{array}$ & $\begin{array}{l}\text { (Lundt } \\
\text { and } \\
\text { Jentschke } \\
\text { 2019) } \\
\end{array}$ & $\begin{array}{l}\text { (Zhang } \\
\text { et al. } \\
2016)\end{array}$ & $\begin{array}{l}\text { (Hojan, } \\
\text { Kwiatkowska- } \\
\text { Borowczyk, et } \\
\text { al. 2016) } \\
\end{array}$ & $\begin{array}{l}\text { (Poort } \\
\text { et al. } \\
2020)\end{array}$ & $\begin{array}{lr}\text { M } & \text { E } \\
\text { Mendoza } \\
\text { et al. } \\
2017) \\
\end{array}$ & $\begin{array}{l}\text { (Charalambous } \\
\text { et al. 2016) }\end{array}$ & $\begin{array}{l}\text { (Johns } \\
\text { et al. } \\
2015)\end{array}$ & Result/mean \\
\hline & Hematologi & 25 & - & 17 & - & 17 & - & 8 & - & - & 4 & 3 & - & - & 74 \\
\hline & $\begin{array}{l}\text { Head, neck, and } \\
\text { neurologi }\end{array}$ & - & - & 28 & - & - & - & 3 & 91 & - & - & 2 & - & - & 124 \\
\hline & $\begin{array}{l}\text { Sistem } \\
\text { pencernaan }\end{array}$ & 21 & - & 11 & - & - & - & 5 & - & - & 34 & - & - & 37 & 108 \\
\hline & System nefrologi & - & - & - & - & - & - & 4 & - & - & 36 & 2 & 104 & - & 146 \\
\hline & Other & 21 & - & - & - & - & - & - & - & - & - & 8 & - & - & 29 \\
\hline & $\begin{array}{l}\text { Cancer Stage } \\
\text { Stage } 0\end{array}$ & 17 & - & - & - & - & - & - & - & - & - & - & - & 10 & 27 \\
\hline & Stage I & 127 & 60 & - & 23 & - & - & - & 3 & - & - & - & - & 21 & 234 \\
\hline & Stage II & 122 & 2 & - & 55 & - & - & - & 8 & - & - & - & - & 10 & 197 \\
\hline & Stage III & 57 & - & - & 9 & - & - & - & 21 & - & - & - & - & 30 & 117 \\
\hline & Stage IV & 10 & 10 & - & - & - & - & - & 59 & - & - & - & - & - & 79 \\
\hline & Unknown & 25 & - & - & - & - & - & - & - & - & - & - & - & - & 25 \\
\hline & $\begin{array}{l}\text { Treatment } \\
\text { Operation }\end{array}$ & - & - & - & - & - & - & - & - & - & - & - & 61 & 10 & 71 \\
\hline & Chemotreatment & - & - & - & - & - & - & - & - & - & 66 & - & 96 & 20 & 182 \\
\hline
\end{tabular}


D Hudiyawati \& W Syafitry / Jurnal Kesehatan 14 (2) 2021, 195-211

\begin{tabular}{|c|c|c|c|c|c|c|c|c|c|c|c|c|c|c|c|}
\hline No & Component & $\begin{array}{l}\text { (Lin } \\
\text { et al. } \\
2019 \text { ) }\end{array}$ & $\begin{array}{l}\text { (Amy J. } \\
\text { Hoffman } \\
\text { et al. } \\
2017)\end{array}$ & $\begin{array}{l}\text { (Schuler } \\
\text { et al. } \\
2017)\end{array}$ & $\begin{array}{l}\text { (Lu } \\
\text { et al. } \\
2019)\end{array}$ & $\begin{array}{l}\text { (Bryant } \\
\text { et al. } \\
2018)\end{array}$ & $\begin{array}{l}\text { (Dhillon } \\
\text { et al. } \\
2017 \text { ) }\end{array}$ & $\begin{array}{l}\text { (Lundt } \\
\text { and } \\
\text { Jentschke } \\
\text { 2019) } \\
\end{array}$ & $\begin{array}{l}\text { (Zhang } \\
\text { et al. } \\
2016)\end{array}$ & $\begin{array}{l}\text { (Hojan, } \\
\text { Kwiatkowska- } \\
\text { Borowczyk, et } \\
\text { al. 2016) }\end{array}$ & $\begin{array}{l}\text { (Poort } \\
\text { et al. } \\
2020 \text { ) }\end{array}$ & $\begin{array}{lr}\text { M } & \text { E } \\
\text { Mendoza } \\
\text { et al. } \\
2017) \\
\end{array}$ & $\begin{array}{l}\text { (Charalambous } \\
\text { et al. 2016) }\end{array}$ & $\begin{array}{l}\text { (Johns } \\
\text { et al. } \\
2015)\end{array}$ & Result/mean \\
\hline & $\begin{array}{l}\text { Radiation } \\
\text { treatment }\end{array}$ & - & - & - & - & - & - & - & - & - & - & - & 51 & 41 & 92 \\
\hline & $\begin{array}{l}\text { Hormonal } \\
\text { treatment }\end{array}$ & - & - & - & - & - & - & - & - & - & 44 & - & - & - & 44 \\
\hline & Immunotreatment & - & - & - & - & - & - & - & - & - & 5 & - & - & - & 5 \\
\hline & $\begin{array}{l}\text { Targeted } \\
\text { treatment }\end{array}$ & - & - & - & - & - & - & - & - & - & 14 & - & - & - & 14 \\
\hline & Drug & - & - & - & - & - & - & - & - & - & 1 & - & - & - & 1 \\
\hline
\end{tabular}

The characteristics of the disease of the study are the type of cancer, stage of cancer, and type of treatment. Types of cancer are gynecology is 560 respondents, hematology is 74 respondents, types of cancer in the head and neck and the neurological system as many as 124 respondents, cancer in the digestive system as many as 108 respondents, cancer in the nephrology system as many as 146 respondents, and other types of cancer as many as 29 respondents. The characteristics of cancer stage are stage 0 is 27 respondents, stage I is 234 respondents, stage II is 197 respondents, stage III is 117 respondents, stage IV is 79 respondents, and unknown is 25 respondents. The characteristics of the type of treatment, including surgery, is 71 respondents; chemo treatment is 182 respondents, radiation treatment is 92 respondents, hormonal treatment is 44 respondents, immune treatment is five respondents, targeted treatment is 14 respondents, drug treatment is one respondent, unknown treatment is two respondents.

The following table is Study Result that include author name and year, study design, participants, duration, instrument and intervention, summary and conclusions for each jurnals. 
D Hudiyawati \& W Syafitry / Jurnal Kesehatan 14 (2) 2021, 195-211

Table 3. Study Result

\begin{tabular}{|c|c|c|c|c|c|c|}
\hline No & $\begin{array}{c}\text { Author and } \\
\text { year }\end{array}$ & Study design & Participants & Duration & Instrumen / Intervention & Summary of results / conclusions \\
\hline 1 & $\begin{array}{c}\text { (Lin et al. } \\
\text { 2019) }\end{array}$ & $\begin{array}{l}\text { A randomized } \\
\text { controlled trial }\end{array}$ & $\begin{array}{c}\text { YOCAS } 177 \\
\text { participants } \\
\text { Standard care } \\
\text { services } 181 \\
\text { participants }\end{array}$ & 4 weeks & $\begin{array}{l}\text { The intervention was } \\
\text { Yoga, and the instruments } \\
\text { used were the } \\
\text { Multidimentional Fatigue } \\
\text { Symptoms Inventory } \\
\text { (MFSI) before and after } \\
\text { the intervention. }\end{array}$ & $\begin{array}{c}\text { YOCAS intervention: Before } \\
\text { intervention }(23.6 \pm 1.6) \text {, after } \\
\text { intervention }(13.4 \pm 1.6) \text {, within- } \\
\text { group difference }(-9.5 \pm 1.2, \mathrm{P} \\
<0.01) \text {. } \\
\text { Standard service maintenance: } \\
\text { Before intervention }(23.8 \pm 1.7), \\
\text { after intervention }(20.2 \pm 1.6), \\
\text { within-group difference }(-2.7 \pm 1.0, \\
\quad \mathrm{P}=0.13) \text {. } \\
\text { Between-group difference }(-6.8 \pm \\
1.4, \mathrm{P}<0.01) \text {. }\end{array}$ \\
\hline 2 & $\begin{array}{l}\text { (Amy J. } \\
\text { Hoffman et al. } \\
\text { 2017) }\end{array}$ & $\begin{array}{c}\text { Randomized } \\
\text { Controlled Trial }\end{array}$ & $\begin{array}{l}\text { The intervention } \\
\text { group (IG) } 37 \\
\text { participants } \\
\text { Control group } \\
\text { (CG) } 35 \\
\text { participants }\end{array}$ & 6 weeks & $\begin{array}{l}\text { The intervention carried } \\
\text { out was Perceived Self- } \\
\text { efficacy. } \\
\text { The instruments used were } \\
\text { the Brief Fatigue Inventory } \\
\text { (BFI) and Perceived Self- } \\
\text { Efficacy for Fatigue Self- } \\
\text { management (PSEFSM). }\end{array}$ & $\begin{array}{l}\text { Before intervention (IG: mean, } 2.2 \\
\text { [SD, 2.0]; CG: mean, } 2.0 \text { [SD, 1.9]; } \\
\text { t70 = 0.44; P = .66; 95\% confidence } \\
\text { interval [CI], 0.73Y1.14). After GI } \\
\text { intervention: mean, 4.1 [SD, 1.9]; } \\
\text { CG: mean, 3.9 [SD, 2.7]; t61 = 0.33; } \\
\text { P = .74; 95\% CI, 0.92Y1.3) for the } \\
\text { IG and CG show no significant } \\
\text { difference. } \\
\text { PSEFSM instrument: Before } \\
\text { intervention (IG: mean, 7.4, CG: } \\
\text { mean, 8.7), After intervention (IG: } \\
\text { mean, 7.0, CG: mean, 7.7). }\end{array}$ \\
\hline 3 & $\begin{array}{c}\text { (Schuler et al. } \\
\text { 2017) }\end{array}$ & $\begin{array}{l}\text { A Randomized } \\
\text { Controlled Trial }\end{array}$ & $\begin{array}{l}\text { Group A (control) } \\
24 \text { patients } \\
\text { Group B (self- } \\
\text { directed) } 23\end{array}$ & 12 weeks & $\begin{array}{l}\text { The intervention carried } \\
\text { out was a Different } \\
\text { Exercise Program. } \\
\text { The instruments used are }\end{array}$ & $\begin{array}{l}\text { Before intervention: General fatigue } \\
\text { (A: mean, } 10.52 \pm 3 \text {, B: mean, } 9.95 \\
\quad \pm 2.95, \mathrm{C}: \text { mean, } 11.59 \pm 3.95) \\
\text { After the intervention: General }\end{array}$ \\
\hline
\end{tabular}


D Hudiyawati \& W Syafitry / Jurnal Kesehatan 14 (2) 2021, 195-211

\begin{tabular}{|c|c|c|c|c|c|c|}
\hline No & $\begin{array}{c}\text { Author and } \\
\text { year }\end{array}$ & Study design & Participants & Duration & Instrumen / Intervention & Summary of results / conclusions \\
\hline & & & $\begin{array}{c}\text { patients } \\
\text { Group C (partially } \\
\text { supervised) } 23 \\
\text { patients }\end{array}$ & & $\begin{array}{l}\text { the Multidimensional } \\
\text { Fatigue Symptoms } \\
\text { Inventory (MFSI) }\end{array}$ & $\begin{array}{c}\text { fatigue }(\mathrm{A} \text { : mean, } 10.81 \pm 3.47, \mathrm{~B}: \\
\text { mean, } 9.40 \pm 4.95, \mathrm{C}: \text { mean, } 10.46 \pm \\
5.14\end{array}$ \\
\hline ,4 & $\begin{array}{l}\text { (Lu et al. } \\
\text { 2019) }\end{array}$ & $\begin{array}{l}\text { A randomized } \\
\text { controlled trial }\end{array}$ & $\begin{array}{l}\text { Baduanjin exercise } \\
\text { group (EG) } 43 \\
\text { patients } \\
\text { Control group } \\
\text { (CG) } 45 \text { patients }\end{array}$ & 24 weeks & $\begin{array}{l}\text { The intervention carried } \\
\text { out was the Baduanjin } \\
\text { qigong exercise. } \\
\text { The instrument used was } \\
\text { the Brief Fatigue Inventory } \\
\text { (BFI). }\end{array}$ & $\begin{array}{c}\text { Before intervention (EG: mild, } 17 \\
\text { (39.5), moderate, } 16 \text { (37.2), severe, } \\
10 \text { (23.3). CG: mild, } 15 \text { (34.1), } \\
\text { moderate, } 17 \text { (38.6), severe, } 12 \\
\text { (27.3). = 0.850) } \\
12 \text { weeks of intervention (EG: mild, } \\
18 \text { (41.9), moderate, } 18 \text { (41.8), } \\
\text { severe, } 7 \text { (16.3). CG: mild, } 17 \\
\text { (38.6), moderate, } 17 \text { (38.6), severe, } \\
10 \text { (22.8). P = 0.750) } \\
24 \text { weeks after intervention (EG: } \\
\text { mild, } 31 \text { (72.1), moderate, } 5(11.6), \\
\text { severe, } 5 \text { (11.6). CG: mild, } 18 \\
\text { (40.9), moderate, } 20 \text { (45.5), severe, } \\
6(13.6) . P<0.01) \text {. }\end{array}$ \\
\hline 5 & $\begin{array}{c}\text { (Bryant et al. } \\
\text { 2018) }\end{array}$ & $\begin{array}{l}\text { randomized } \\
\text { clinical trial }\end{array}$ & $\begin{array}{l}\text { Intervention group } \\
\text { (IG) } 8 \text { patients } \\
\text { Control group } \\
\text { (CG) } 9 \text { patients }\end{array}$ & 4 weeks & $\begin{array}{l}\text { The intervention carried } \\
\text { out was the Effect of } \\
\text { Exercise Performance- } \\
\text { Based Physical Funtion. }\end{array}$ & $\begin{array}{l}\text { Before intervention (IG: 57, EG: } \\
\text { 51.5), after intervention (IG: 50.4, } \\
\text { EG: 55.6), change (IG: -5.95, EG: } \\
\text { 4.1, P=0.11). }\end{array}$ \\
\hline 6 & $\begin{array}{c}\text { (Dhillon et al. } \\
\text { 2017) }\end{array}$ & $\begin{array}{l}\text { A randomized } \\
\text { controlled trial }\end{array}$ & $\begin{array}{c}\text { Exercise group } \\
\text { (EX) } 56 \text { patients, } \\
\text { usual care (UC) } 55 \\
\text { patients }\end{array}$ & 8 months & $\begin{array}{l}\text { The intervention carried } \\
\text { out is Physical Activity. } \\
\text { The instrument used was } \\
\text { the Functional Assessment } \\
\text { of Cancer Treatment- } \\
\text { Fatigue (FACT-F) } \\
\text { subscale. }\end{array}$ & $\begin{array}{l}\text { Before intervention (EX: } 38.43 \text {, UC: } \\
\text { 36.34), } 2 \text { months of intervention } \\
\text { (EX: } 37.53 \text {, UC: } 36.36 \text {, Estimate } \\
\text { 1.17, CI: }-3.46,5.80, P=0.618 \text { ), } 4 \\
\text { months of intervention (EX: } 39.38 \text {, } \\
\text { UC: } 35.33 \text {, Estimate } 4.05 \text {, CI: }-0.88 \text {, } \\
\text { 8.97, P = 0.107), and after } 6 \text { months } \\
\text { of intervention (EX: } 36.67 \text {, UC: }\end{array}$ \\
\hline
\end{tabular}


D Hudiyawati \& W Syafitry / Jurnal Kesehatan 14 (2) 2021, 195-211

\begin{tabular}{|c|c|c|c|c|c|c|}
\hline No & $\begin{array}{l}\text { Author and } \\
\text { year }\end{array}$ & Study design & Participants & Duration & Instrumen / Intervention & Summary of results / conclusions \\
\hline & & & & & & $\begin{array}{c}\text { 34.00, Estimate } 2.67, \mathrm{CI}:-2.58 \\
7.92, \mathrm{P}=0.317)\end{array}$ \\
\hline 7 & $\begin{array}{l}\text { (Lundt and } \\
\text { Jentschke } \\
\text { 2019) }\end{array}$ & $\begin{array}{l}\text { a randomized } \\
\text { controlled study }\end{array}$ & Total 58 patients & 6 months & $\begin{array}{l}\text { The intervention } \\
\text { undertaken is Yoga. } \\
\text { The instrument used was } \\
\text { the EORTC QLQ-FA13 } \\
\text { (European Organization } \\
\text { for Research and } \\
\text { Treatment of Cancer } \\
\text { Quality of Life } \\
\text { Questionnaire-Fatigue } \\
\text { Scale). }\end{array}$ & $\begin{array}{l}\text { Before and after the intervention (n: } \\
\text { 58, mean: } 28.30 \text {, SD: } 8.29 \text {, mean: } \\
\text { 25.69, SD: } 8.03 \text { P }=0.006 \text {, SES: - } \\
\text { 0.31, CI: }-0.54 \text { to }-0.09) \text {. }\end{array}$ \\
\hline 8 & $\begin{array}{l}\text { (Zhang et al. } \\
\text { 2016) }\end{array}$ & $\begin{array}{l}\text { A randomized } \\
\text { controlled trial }\end{array}$ & $\begin{array}{c}\text { Tai Chi } \\
\text { intervention group } \\
\text { (TC) } 47 \text { patients, } \\
\text { Control group } \\
\text { (CG) } 44 \text { patients }\end{array}$ & 2 months & $\begin{array}{l}\text { The intervention } \\
\text { undertaken was Tai chi. } \\
\text { The instrument used was } \\
\text { the Multidimentional } \\
\text { Fatigue Symptoms } \\
\text { Inventory (MFSI). }\end{array}$ & $\begin{array}{l}\text { Before intervention (TC mean (SD): } \\
\text { 46.0 (11.6), CG mean (SD): } 46.8 \\
\text { (12.2), } 6 \text { weeks of intervention (TC } \\
\text { mean (SD): } 59.5 \text { (11.3), CG mean } \\
\text { (SD): } 66.8 \text { (11.9), after } 12 \text { weeks of } \\
\text { intervention (TC mean (SD): } 53.3 \\
\text { (11.8), CG mean (SD): } 59.3(12.2) \text {, } \\
\text { P <0.05). }\end{array}$ \\
\hline 9 & $\begin{array}{l}\quad \text { (Hojan, } \\
\text { Kwiatkowska- } \\
\text { Borowczyk, et } \\
\quad \text { al. 2016) }\end{array}$ & $\begin{array}{l}\text { A randomized } \\
\text { clinical study }\end{array}$ & $\begin{array}{l}\text { Intervention group } \\
\text { (EG) } 27 \text { patients } \\
\text { Usual group (UG) } \\
27 \text { patients }\end{array}$ & 8 weeks & $\begin{array}{c}\text { The intervention carried } \\
\text { out was Physical Exercise. } \\
\text { The instrument used was } \\
\text { The Functional } \\
\text { Assessment of Cancer } \\
\text { Treatment-Fatigue (FACT- } \\
\text { F). }\end{array}$ & $\begin{array}{c}\text { Before intervention (EG mean (SD) } \\
27.3 \text { (19.7), UG mean (SD): } 28.0 \\
(21.9) \text { ), after intervention (EG mean } \\
\text { (SD): } 30.7(21.4) \text {, UG mean (SD): } \\
242.1(23.6), \text { mean } 3.4(19.3), \text { P } \\
<0.05) .\end{array}$ \\
\hline 10 & $\begin{array}{l}\text { (Poort et al. } \\
\text { 2020) }\end{array}$ & $\begin{array}{l}\text { a randomized } \\
\text { controlled trial }\end{array}$ & $\begin{array}{l}\text { CBT group } 46 \\
\text { patients, GET } \\
\text { group } 42 \text { patients, } \\
\text { and usual care } \\
\end{array}$ & 26 weeks & $\begin{array}{l}\text { The intervention carried } \\
\text { out was CBT. } \\
\text { The instrument used was } \\
\text { (EORTC-QLQ-C30 or }\end{array}$ & $\begin{array}{l}\text { CBT significantly reduced fatigue at } \\
14 \text { weeks compared with usual care } \\
{[-7.2,97.5 \% \text { confidence interval }} \\
\text { (CI) }-12.7 \text { to }-1.7 ; \mathrm{P}^{1 / 4} 0.003, \mathrm{~d}^{1 / 4}\end{array}$ \\
\hline
\end{tabular}


D Hudiyawati \& W Syafitry / Jurnal Kesehatan 14 (2) 2021, 195-211

\begin{tabular}{|c|c|c|c|c|c|c|}
\hline No & $\begin{array}{c}\text { Author and } \\
\text { year }\end{array}$ & Study design & Participants & Duration & Instrumen / Intervention & Summary of results / conclusions \\
\hline & & & $\begin{array}{c}\text { group (UC) } 46 \\
\text { patients }\end{array}$ & & SIP8) as covariates. & $\begin{array}{l}\text { 0.7]. (UC: mean } 38.95 \text { (35.58 to } \\
\text { 42.32), CBT: mean } 31.72 \text { (28.36 to } \\
\text { 35.09), GET: mean } 34.25 \text { (30.79 to } \\
\text { 37.71, } \mathrm{P}=0.012) \text {. }\end{array}$ \\
\hline 11 & $\begin{array}{l}\text { (M E Mendoza } \\
\text { et al. 2017) }\end{array}$ & $\begin{array}{l}\text { A randomized } \\
\text { controlled trial }\end{array}$ & $\begin{array}{l}\text { CBT intervention } \\
\text { group } 22 \text { patients, } \\
\text { EC intervention } \\
\text { group } 22 \text { patients }\end{array}$ & 3 method & $\begin{array}{c}\text { The intervention } \\
\text { undertaken is Hypnosis } \\
\text { plus CBT. } \\
\text { The instrument used was } \\
\text { the Patient-reported } \\
\text { Outcomes Measurement } \\
\text { Information System } \\
\text { (PROMIS). }\end{array}$ & $\begin{array}{l}\text { In pretreatment to post-treatment } \\
\text { changes in the primary outcomes, } \\
\text { found significantly greater } \\
\text { improvements }(\mathrm{P}<.001) \text { significant } \\
\text { between-groups differences emerged } \\
\text { for depression }(\mathrm{P}<.001) \text {, cancer } \\
\text { distress }(\mathrm{P}<.001) \text {, pain interference } \\
(\mathrm{P}<.05) \text {, and pain catastrophizing } \\
(\mathrm{P}<.05) \text {. }\end{array}$ \\
\hline 12 & $\begin{array}{c}\text { (Charalambous } \\
\text { et al. 2016) }\end{array}$ & $\begin{array}{l}\text { A randomized } \\
\text { controlled trial }\end{array}$ & $\begin{array}{l}\text { Intervention group } \\
\text { (IG) } 104 \text { patients, } \\
\text { control group } \\
\text { (CG) } 104 \text { patients }\end{array}$ & 4 weeks & $\begin{array}{l}\text { The interventions carried } \\
\text { out were Guided Imagery } \\
\text { and Progressive Muscle } \\
\text { Relaxation. } \\
\text { The instrument used is The } \\
\text { Cancer Fatigue Scale } \\
\text { (CFS) }\end{array}$ & $\begin{array}{l}\text { Before intervention (IG: mean 67.8 } \\
\text { (19.6), mean CG 73.1 (21.8). } \\
\text { After intervention (IG: mean -17.1, } \\
\text { P <0.00001), and (CG: mean }+7.6 \text {, P } \\
<0.00001) .\end{array}$ \\
\hline 13 & $\begin{array}{c}\text { (Johns et al. } \\
2015)\end{array}$ & $\begin{array}{l}\text { A randomized } \\
\text { controlled trial }\end{array}$ & $\begin{array}{l}\text { The MBSR group } \\
\text { was } 35 \text { patients, } \\
\text { the education } \\
\text { support (ES) } \\
\text { group was } 36 \\
\text { patients }\end{array}$ & 6 months & $\begin{array}{l}\text { The intervention } \\
\text { undertaken is Mindfulness- } \\
\text { based Stress Reduction. } \\
\text { The instrument used is The } \\
\text { Attentional Function Index } \\
\text { (AFI). }\end{array}$ & $\begin{array}{c}\text { No differences emerged between } \\
\text { MBSR and ES participants at T2 or } \\
\text { T3 (all } \mathrm{p}>0.64 \text { ) on Stroop } \\
\text { interference scores. between T1 and } \\
\text { T2 }(\mathrm{p}=0.17) \text {, MBSR participants had } \\
\text { a lower error rate at T3 } \\
\text { (MT3=-0.01, SDT3=0.03) relative } \\
\text { to T1 }(\mathrm{MT} 1=-0.10, \mathrm{SDT} 1=0.25 \\
\mathrm{z}=-2.12, \mathrm{n}=29, \mathrm{p}=0.034, \mathrm{r}=0.39)\end{array}$ \\
\hline
\end{tabular}




\section{Physical treatment}

Physical treatment is an exercise program such as walking, cycling, or running (Schuler et al. 2017). Exercise program for walking and balance training, a program of walking for 30 minutes, heating phase for 5 minutes, brisk walking for 10 minutes, and cool down for 5 minutes are effective in reducing cancer-related fatigue (Amy J Hoffman et al. 2017). Aerobic exercise program and body resistance training (Bryant et al. 2018). Thirty minutes of aerobic exercise (brisk walking, treadmill, or using a bicycle) and 15 minutes of resistance training, research shows that a combination of aerobic exercise and resistance training is effective in reducing fatigue during treatment in a variety of cancer treatments (Hojan, KowskaBorowczyk, et al. 2016).

Cancer-specific gymnastics education program (move your body), and nutrition (eat for health) (Dhillon et al. 2017). Baduanjin qigong exercise is the first 40 minutes of explaining and demonstrating eight movements and natural breathing methods, and patients practice Baduanjin for five sessions per week for 20-40 minutes ( $\mathrm{Lu}$ et al. 2019). Tai chi exercises with easy movements is 1) initial form (hands rising to shoulder level), 2) arms arching back, 3) stepping sideways and moving arms, 4) moving hands, 5) diagonal steps, 6) standing with one leg, 7) stepping and pushing, and 8) the form of a cover (hands fall sideways, left foot pulled to right foot). Each session includes 5 to 10 minutes of warmup, followed by a Tai Chi workout in a practice session (Zhang et al. 2016).

The effect of reducing fatigue on physical exercise was found in the intervention group compared to the control group. Although this study looked at the impact of physical activity in both the intervention and control groups, a significant change from severe fatigue could only be found in the intervention group. This study indicates that severe fatigue in cancer patients reduced by performing appropriate physical exercise (Schuler et al. 2017). The results of Hoffman's (2017) study are very satisfying because the intervention group found the intervention very acceptable, " fun, " " convenient for exercising at home, " " easy to use, ". When compared with the control group, the intervention group showed initial efficacy in reducing the severity of CRF every week for six weeks of intervention (Amy J Hoffman et al. 2017). Other studies have shown that a combination of aerobic exercise and resistance training is effective in reducing fatigue during treatment in a variety of cancer treatments (Bryant et al. 2018; Dhillon et al. 2017; Hojan, KowskaBorowczyk, et al. 2016).

This study showed a positive effect in reducing CRF and improving sleep quality. Baduanjin relieved CRF in colorectal cancer patients undergoing chemo treatment. convenient and straightforward method of lowering CRF in patients with colorectal cancer who are undergoing chemo treatment ( $\mathrm{Lu}$ et al. 2019). The results show that Tai Chi is an effective intervention for managing fatigue in pulmonary patients undergoing chemo treatment, significantly to reduce fatigue and increase strength (Zhang et al. 2016). The physical exercise intervention was significantly effective at reducing CRF during and after cancer treatment (Mustian et al. 2017). The results suggest exercising during chemo treatment is a promising strategy for minimizing treatment-related side effects, both short and long term (Witlox et al. 2018). 


\section{Psychological treatment}

Yoga treatment 60 minutes each week for eight weeks (Lundt and Jentschke 2019). Yoga consists of breathing exercises, physical alignment postures, and mindfulness exercises, sessions lasting 75 minutes per week for four weeks (Lin et al. 2019). CBT (Cognitive Behavior Treatment) for 1 hour in 12 weeks, and GET (Guided Exercise Treatment) exercises consist of an aerobic exercise program for 2 hours in 12 weeks (Poort et al. 2020). Programs combine self-hypnosis training with CBT (M. E. Mendoza et al. 2017).

A two-minute breathing exercise intervention, followed by a 10-minute progressive muscle relaxation exercise and a 15-minute guided imagery session. Progressive Muscle Relaxation. Progressive Muscle Relaxation is a nursing intervention of the Nursing Intervention Classification (NIC), is defined as facilitating the successive tension and release of muscle groups while noticing the resulting difference in sensation over four weeks (Charalambous et al. 2016). The MBSR (Mindfulnessbased stress reduction) and ES (Education Support) interventions consist of twohour classes each per week led by a skilled facilitator following standard procedures (Johns et al. 2015).

Research shows that Hatha-based and Restorative yoga treatment is effective for treating CRF in people with cancer. The results also indicated that $22 \%$ of the YOCAS effect on CRF was associated with improved overall sleep quality (Lin et al. 2019). The results showed that yoga exhibited moderate to large effect sizes in reducing fatigue compared to conventional treatments (Hilfiker et al. 2018).

The Guided Imagery and Progressive Muscle Relaxation intervention, showing a statistically significant reduction in fatigue felt after the intervention, data analysis revealed a statistically significant decrease in perceived fatigue after the intervention. This decrease reflected in the physical, affective, and cognitive subscales of the cancer fatigue scale (Charalambous et al. 2016). The Guided Imagery and Progressive Muscle Relaxation intervention, showing a statistically significant reduction in fatigue felt after the intervention, data analysis revealed a statistically significant decrease in perceived fatigue after the intervention. This decrease reflected in the physical, affective, and cognitive subscales of the cancer fatigue scale (Johns et al. 2015). Small to moderate effect sizes on reducing fatigue found for the CBT combination (Hilfiker et al. 2018). Psychological interventions were significantly effective at reducing CRF during and after cancer treatment (Mustian et al. 2017).

\section{CONCLUSION}

The type of physical treatment that is most effective in reducing symptoms of fatigue is the combination of resistance training and aerobic exercise. The effect of physical treatment can reduce signs of fatigue in cancer patients during and after treatment. Some interventions are physical treatment and psychological treatment, and physical treatment includes Different Exercise programs, Perceived Self-efficacy, Physical Activity, aerobics, Physical Exercise, Exercise Behaviors, Walking Exercise Program, Baduanjin qigong exercise, and thai chi.

The type of psychological treatment most effective in reducing symptoms of fatigue is Yoga for Cancer Survivors. Psychological treatment can also reduce signs of fatigue in cancer patients during and after treatment. Several interventions are Yoga, YOCAS (The Yoga for Cancer Survivors), Cognitive-behavioral treatment or graded exercise treatment 
compared with usual care, Hypnosis plus CBT, Guided Imagery, and Progressive Muscle. Relaxation, Mindfulness-based stress reduction.

\section{ACKNOWLEDGMENT}

The authors would like to thank the support of anonymous reviewer for their suggestion to revise this paper.

\section{REFERENCES}

Aapro, Matti, Florian Scotte, Thierry Bouillet, David Currow, and Antonio Vigano. (2017). "A Practical Approach to Fatigue Management in Colorectal Cancer." Clinical Colorectal Cancer 16(4):275-85.

Akaza, Hideyuki. (2019). "International Agency for Research on Cancer (IARC)." Japanese Journal of Cancer and Chemotherapy 46(1):34-35.

Bryant, Ashley Leak, Allison M. Deal, Claudio L. Battaglini, Brett Phillips, Mackenzi Pergolotti, Erin Coffman, Matthew C. Foster, William A. Wood, Charlotte Bailey, Anthony C. Hackney, Deborah K. Mayer, Hyman B. Muss, and Bryce B. Reeve. (2018). "The Effects of Exercise on Patient-Reported Outcomes and PerformanceBased Physical Function in Adults With Acute Leukemia Undergoing Induction Therapy: Exercise and Quality of Life in Acute Leukemia (EQUAL)." Integrative Cancer Therapies 17(2):263-70.

Charalambous, Andreas, Ann M. Berger, Ellyn Matthews, Dave D. Balachandran, Evridiki Papastavrou, and Oxana Palesh. (2019). "Cancer-Related Fatigue and Sleep Deficiency in Cancer Care Continuum: Concepts, Assessment, Clusters, and Management." Supportive Care in Cancer: Official Journal of the Multinational Association of Supportive Care in Cancer 27(7):2747-53.

Charalambous, Andreas, Margarita Giannakopoulou, Evaggelos Bozas, Yiola Marcou, Petros Kitsios, and Lefkios Paikousis. (2016). "Guided Imagery and Progressive Muscle Relaxation as a Cluster of Symptoms Management Intervention in Patients Receiving Chemotherapy: A Randomized Control Trial." PLoS ONE 11(6):1-18.

Dhillon, H. M., M. L. Bell, H. P. van der Ploeg, J. D. Turner, M. Kabourakis, L. Spencer, C. Lewis, R. Hui, P. Blinman, S. J. Clarke, M. J. Boyer, and J. L. Vardy. (2017). "Impact of Physical Activity on Fatigue and Quality of Life in People with Advanced Lung Cancer: A Randomized Controlled Trial." Annals of Oncology: Official Journal of the European Society for Medical Oncology 28(8):1889-97.

Higgins, Julian P., James Thomas, Jacqueline Chandler, Miranda Cumpston, Tianjing Li, Matthew Page J, and Vivian A. Welch. (2019). Cochrane Handbook for Systematic Reviews of Interventions. Second Edi. USA: The Cochrane Collaboration.

Hilfiker, Roger, Andre Meichtry, Manuela Eicher, Lina Nilsson Balfe, Ruud H. Knols, 
Martin L. Verra, and Jan Taeymans. (2018). "Exercise and Other NonPharmaceutical Interventions for Cancer-Related Fatigue in Patients during or after Cancer Treatment: A Systematic Review Incorporating an IndirectComparisons Meta-Analysis." British Journal of Sports Medicine 52(10):651-58.

Hoffman, Amy J, Ruth Ann Brintnall, Barbara A. Given, Alexander von Eye, Lee W. Jones, and Jean K. Brown. (2017). "Using Perceived Self-Efficacy to Improve Fatigue and Fatigability In Postsurgical Lung Cancer Patients: A Pilot Randomized Controlled Trial." Cancer Nursing 40(1):1-12.

Hoffman, Amy J., Ruth Ann Brintnall, Barbara A. Given, Alexander Von Eye, Lee W. Jones, and Jean K. Brown. (2017). "Using Perceived Self-Efficacy to Improve Fatigue and Fatigability in Postsurgical Lung Cancer Patients: A Pilot Randomized Controlled Trial.” Cancer Nursing 40(1):1-12.

Hojan, Katarzyna, Eliza Kwiat Kowska-Borowczyk, Ewa Leporowska, MacIej Gorecki, Owidia Ozga-Majchrzak, Tomasz Milecki, and Piotr Milecki. (2016). "Physical Exercise for Functional Capacity, Blood Immune Function, Fatigue, and Quality of Life in High-Risk Prostate Cancer Patients during Radiotherapy: A Prospective, Randomized Clinical Study." European Journal of Physical and Rehabilitation Medicine 52(4):489-501.

Hojan, Katarzyna, Eliza Kwiatkowska-Borowczyk, Ewa Leporowska, Maciej Górecki, Owidia Ozga-Majchrzak, Tomasz Milecki, and Piotr Milecki. (2016). "Physical Exercise for Functional Capacity, Blood Immune Function, Fatigue, and Quality of Life in High-Risk Prostate Cancer Patients during Radiotherapy: A Prospective, Randomized Clinical Study." European Journal of Physical and Rehabilitation Medicine 52(4):489-501.

Johns, Shelley A., Linda F. Brown, Kathleen Beck-Coon, Patrick O. Monahan, Yan Tong, and Kurt Kroenke. (2015). "Randomized Controlled Pilot Study of Mindfulness-Based Stress Reduction for Persistently Fatigued Cancer Survivors." Psycho-Oncology 24(8):885-93.

Lin, Po-Ju, Ian R. Kleckner, Kah Poh Loh, Julia E. Inglis, Luke J. Peppone, Michelle C. Janelsins, Charles S. Kamen, Charles E. Heckler, Eva Culakova, Wilfred R. Pigeon, Pavan S. Reddy, Michael J. Messino, Rakesh Gaur, and Karen M. Mustian. (2019). "Influence of Yoga on Cancer-Related Fatigue and on Mediational Relationships Between Changes in Sleep and Cancer-Related Fatigue: A Nationwide, Multicenter Randomized Controlled Trial of Yoga in Cancer Survivors.” Integrative Cancer Therapies 18:1534735419855134.

Lu, Yun, Hui-Qin Qu, Feng-Ying Chen, Xiao-Ting Li, Lan Cai, Shan Chen, and YuanYuan Sun. (2019). "Effect of Baduanjin Qigong Exercise on Cancer-Related Fatigue in Patients with Colorectal Cancer Undergoing Chemotherapy: A Randomized Controlled Trial." Oncology Research and Treatment 42(9):431-39.

Lundt, Anna, and Elisabeth Jentschke. (2019). "Long-Term Changes of Symptoms of Anxiety, Depression, and Fatigue in Cancer Patients 6 Months After the End of 
Yoga Therapy." Integrative Cancer Therapies 18:1534735418822096.

Mendoza, M E, A. Capafons, J. R. Gralow, K. L. Syrjala, J. M. Suárez-Rodríguez, J. R. Fann, and M. P. Jensen. (2017). "Randomized Controlled Trial of the Valencia Model of Waking Hypnosis plus CBT for Pain, Fatigue, and Sleep Management in Patients with Cancer and Cancer Survivors." Psycho-Oncology 26(11):183238.

Mendoza, M. E., A. Capafons, J. R. Gralow, K. L. Syrjala, J. M. Suárez-Rodríguez, J. R. Fann, and M. P. Jensen. (2017). "Randomized Controlled Trial of the Valencia Model of Waking Hypnosis plus CBT for Pain, Fatigue, and Sleep Management in Patients with Cancer and Cancer Survivors." Psycho-Oncology 26(11):1832-38.

Mustian, Karen M., Catherine M. Alfano, Charles Heckler, Amber S. Kleckner, Ian R. Kleckner, Corinne R. Leach, David Mohr, Oxana G. Palesh, Luke J. Peppone, Barbara F. Piper, John Scarpato, Tenbroeck Smith, Lisa K. Sprod, and Suzanne M. Miller. (2017). "Comparison of Pharmaceutical, Psychological, and Exercise Treatments for Cancer-Related Fatigue: A Meta-Analysis." JAMA Oncology 3(7):961-68.

Poort, H., M. E. W. J. Peters, W. T. A. van der Graaf, P. T. Nieuwkerk, A. J. van de Wouw, M. W. G. Nijhuis-van der Sanden, G. Bleijenberg, C. A. H. H. V. M. Verhagen, and H. Knoop. (2020). "Cognitive Behavioral Therapy or Graded Exercise Therapy Compared with Usual Care for Severe Fatigue in Patients with Advanced Cancer during Treatment: A Randomized Controlled Trial." Annals of Oncology 31(1):115-22.

Riskesdas, Kemenkes. (2018). "Hasil Utama Riset Kesehata Dasar (RISKESDAS)." Journal of Physics A: Mathematical and Theoretical 44(8):1-200.

Saaiq, Muhammad, and Bushra Ashraf. (2017). 'Modifying 'Pico' Question into 'Picos' Model for More Robust and Reproducible Presentation of the Methodology Employed in A Scientific Study.” World Journal of Plastic Surgery 6(3):390-92.

Schuler, Markus K., Leopold Hentschel, Wadim Kisel, Michael Kramer, Felicitas Lenz, Beate Hornemann, Julia Hoffmann, Stephan Richter, Gerhard Ehninger, Martin Bornhäuser, and Frank Kroschinsky. (2017). "Impact of Different Exercise Programs on Severe Fatigue in Patients Undergoing Anticancer TreatmentA Randomized Controlled Trial." Journal of Pain and Symptom Management 53(1):57-66.

Witlox, Lenja, Anouk E. Hiensch, Miranda J. Velthuis, Charlotte N. Steins Bisschop, Maartje Los, Frans L. G. Erdkamp, Haiko J. Bloemendal, Marlies Verhaar, Daan Ten Bokkel Huinink, Elsken van der Wall, Petra H. M. Peeters, Anne M. May, Charles Heckler; Amber S. Kleckner Karen M. Mustian, Catherine M. Alfano, Roger Hilfiker, Andre Meichtry, Manuela Eicher, Lina Nilsson Balfe, Ruud H. Knols, Martin L. Verra, and Jan Taeymans. (2018). "Four-Year Effects of Exercise on Fatigue and Physical Activity in Patients with Cancer." $B M C$ 
D Hudiyawati \& W Syafitry / Jurnal Kesehatan 14 (2) 2021, 195-211

Medicine 16(1):86.

Zhang, Li-Li, Su-Zhen Wang, Hong-Lin Chen, and A. Zhen Yuan. (2016). "Tai Chi Exercise for Cancer-Related Fatigue in Patients With Lung Cancer Undergoing Chemotherapy: A Randomized Controlled Trial." Journal of Pain and Symptom Management 51(3):504-11.

Zohuri, Bahman, and Masoud Moghaddam. (2017). "Business Resilience System (BRS): Driven through Boolean, Fuzzy Logics and Cloud Computation: Real and near Real Time Analysis and Decision Making System." Business Resilience System (BRS): Driven Through Boolean, Fuzzy Logics and Cloud Computation: Real and Near Real Time Analysis and Decision Making System (September 2018):1-425. 